\title{
BMJ Open Attrition from specialised rehabilitation associated with an elevated mortality risk: results from a vital status tracing study in Swiss spinal cord injured patients
}

To cite: Chamberlain JD, Eriks-Hoogland IE, Hug K, et al. Attrition from specialised rehabilitation associated with an elevated mortality risk: results from a vital status tracing study in Swiss spinal cord injured patients. BMJ Open 2020;10:e035752. doi:10.1136/ bmjopen-2019-035752

- Prepublication history and additional material for this paper are available online. To view these files, please visit the journal online (http://dx.doi. org/10.1136/bmjopen-2019035752).

Received 14 November 2019 Revised 01 April 2020 Accepted 28 May 2020

D) Check for updates

(c) Author(s) (or their employer(s)) 2020. Re-use permitted under CC BY-NC. No commercial re-use. See rights and permissions. Published by BMJ.

For numbered affiliations see end of article.

Correspondence to Dr Jonviea D Chamberlain; jonviea.chamberlain@ubordeaux.fr

\section{ABSTRACT}

Introduction Study drop-out and attrition from treating clinics is common among persons with chronic health conditions. However, if attrition is associated with adverse health outcomes, it may bias or mislead inferences for health policy and resource allocation.

Methods This retrospective cohort study uses data attained through the Swiss Spinal Cord Injury (SwiSCl) cohort study on persons with spinal cord injury (SCl). Vital status (VS) was ascertained either through clinic medical records (MRs) or through municipalities in a secondary tracing effort. Flexible parametric survival models were used to investigate risk factors for going lost to clinic (LTC) and the association of LTC with subsequent risk of mortality.

Results 1924 individuals were included in the tracing study; for 1608 of these cases, contemporary VS was initially checked in the MRs. VS was ascertained for 704 cases of the 1608 cases initially checked in MRs; of the remaining cases $(n=904)$, nearly $90 \%$ were identified in municipalities $(\mathrm{n}=804)$. LTC was associated with a nearly fourfold higher risk of mortality ( $\mathrm{HR}=3.62 ; 95 \% \mathrm{Cl} 2.18$ to 6.02) among persons with traumatic $\mathrm{SCl}$. Extended driving time (ie, less than 30 min compared with $30 \mathrm{~min}$ and longer to reach the nearest specialised rehabilitation facility) was associated with an increased risk of mortality ( $\mathrm{HR}=1.51,95 \% \mathrm{Cl} 1.02$ to 2.22$)$ for individuals with nontraumatic SCl.

Conclusion The differential risk of LTC according to sociodemographic and $\mathrm{SCl}$ lesion characteristics underscores the importance of accounting for attrition in cohort studies on chronic disease populations requiring long-term care. In addition, given the associated risk of mortality, LTC is an issue of concern to clinicians and policy makers aiming to optimise the long-term survival of community-dwelling individuals with traumatic $\mathrm{SCl}$. Future studies are necessary to verify whether it is possible to improve survival prospects of individuals LTC through more persistent outreach and targeted care.

\section{INTRODUCTION}

Clinic attrition-or loss to clinic (LTC), in which case individuals experience irregular

\section{Strengths and limitations of this study}

- An extensive tracing effort was undertaken to ascertain the vital status of all individuals lost to clinic in the Swiss rehabilitation setting.

- Only three of the five specialised rehabilitation centres underwent an initial check of medical records for updated vital status information.

- Sensitivity analyses were employed to ensure that systematic differences in tracing methodology across specialised rehabilitation centres did not affect conclusions.

- It was not possible to include secondary health conditions-a potential unmeasured confounder-in the present study.

- E-values were estimated to assess the potential influence of unmeasured confounding on study results and conclusions.

or discontinued care-and study drop-out is common among persons with chronic health conditions, particularly given the necessitated extended follow-up periods to ensure optimal health. ${ }^{1}$ Unfortunately, if an individual's risk of adverse health outcomes is associated with irregular or discontinued care, individuals who go LTC may subsequently be at a higher risk for premature mortality and adverse health outcomes. For example, a follow-up study on attrition among persons with HIV estimated attrition to underestimate 1-year mortality by up to $76 \%{ }^{2}$ An increased propensity for study drop-out or attrition could be indicative of individuals LTC being in poorer health. ${ }^{1}$ The opposite is also conceivable, with individuals in better health more likely to forego scheduled follow-up care. In either case, attrition may bias the available information with respect to health states and healthcare needs of patients and mislead inferences 
for health policy and resource allocation. ${ }^{3}$ However, while evidence on the influence of clinic attrition on health outcomes is available for certain chronic conditions, such as HIV or cancer, in the context of physical impairment evidence is lacking.

Spinal cord injuries (SCIs) are a chronic health condition with life-long repercussions on social and health outcomes. ${ }^{4}$ Unfortunately, with the exception of settings allowing personalised linkage to a national vital statistics database by common person ID (eg, Norway and Australia), most SCI-specific studies have largely ignored the issue of attrition in the estimation of mortality and survival. ${ }^{5-7}$ Studies have either not addressed whether attrition poses an issue to the interpretation of results; not addressed how study attrition was accounted for to reduce potential bias in estimates of mortality and survival; or only addressed it through a narrative account as part of a limitations discussion. The Swiss Spinal Cord Injury (SwiSCI) cohort study is a multicentre, longitudinal study and a rich resource for studies aimed at improving the lives of persons with SCI. However, given that the centres from which the initial data are acquired rely on routine practice for follow-up (ie, passive follow-up, in which centres rely on patients reaching out to inform regarding health), SwiSCI-based studies may be at risk for attrition bias. To resolve this, an extended effort was undertaken to update the database for all study participants without a recent vital status by employing a tracing methodology used previously in the Swiss context. ${ }^{8}$ To investigate the extent and potential impact of attrition within the SwiSCI database, the present study therefore aims to identify relevant risk factors for clinic attrition and to evaluate the potential differential risk in mortality between those LTC compared with those with continued care in Swiss-based SCI-specialised rehabilitation centres.

\section{METHODS}

\section{Tracing methodology}

The Medical Records study, encompassed within the SwiSCI cohort study (described in detail elsewhere), is a retrospective cohort study of persons admitted for first rehabilitation in Switzerland in one of the five specialised rehabilitation centres. ${ }^{9-11}$ Using the Medical Records study as an initial base to identify study participants, the community survey was designed to then follow-up community-dwelling persons with SCI in Switzerland using a prospective panel study design with recurring surveys every 5years. ${ }^{12}$ In September 2011, the first community survey (2012 CS) was sent to potential participants. This provided a natural point at which to re-evaluate and update the vital status (VS) of many individuals identified in the original Medical Records study, based on either active participation or active refusal in the $2012 \mathrm{CS}^{13}{ }^{13}$ For individuals who did not actively respond to the $2012 \mathrm{CS}$, or for whom no recent contact (ie, postSeptember 2011) was made with the study centre, VS was manually updated through a secondary tracing study (figure 1). Briefly, medical records were first checked in specialised rehabilitation centres to determine the last date of contact with the clinic. If an individual had visited the clinic on or after 30 September 2011, they were considered alive with vital status ascertained in clinic and thereby not lost to clinic. If an individual did not have a recent clinic contact, the municipality of their last known address was contacted with a request for information on the VS of the individual. If vital status could be ascertained from municipality records, individuals were considered LTC but not lost to follow-up (LTFU) (note: individuals were only considered LTC if medical records were first checked for VS). Finally, if VS could not be ascertained from municipality records, only then were individuals considered LTFU. The Childhood Cancer Registry previously employed a similar methodology in Switzerland. ${ }^{8}$ Given the integral role of the 2012 CS for ascertaining VS, 30 September 2011 marks the cut-off date for the present study. To ensure adequate data quality, the study was further restricted to individuals who started first rehabilitation from 1990 onwards, when two specialized rehabilitation facilities were opened. Ascertainment of pre-1990 cases was frequently incomplete, partly related to the between-clinic transfer or even destruction of MRs on closure of a historical facility.

From 1990 to 2007, there was no official, operational guideline for the long-term follow-up care of persons living with SCI in Switzerland. However, there was a consensus among physicians in specialised SCI centres that all persons needed regular follow-up visits at 3 months, 6 months, 1 year and then annually. Since 2007, this follow-up scheme has been endorsed as formal guideline by the Swiss Society of Paraplegia and implemented by all clinics for specialised SCI care in Switzerland. ${ }^{14}$ Patients who do not respond to the invitation for a follow-up visit are reminded at least once (by written letter or telephone call) and asked to contact the clinic. Unfortunately, no formal and comprehensive evaluation exists regarding the practical implementation and effectiveness of follow-up procedures.

\section{Descriptive statistics}

For categorical variables, descriptive analyses consisted of crude numbers and percentages; for continuous variables the mean, median, SD and range are reported. Descriptive analyses of non-traumatic SCI (NTSCI) and traumatic SCI (TSCI) are reported stratified by key demographic characteristics and SCI-specific characteristics (ie, stratified by sex, age, lesion level, lesion completeness or time period since injury).$^{15}$ Non-parametric or parametric tests, depending on the distribution, were used to compare group characteristics. Only individuals who were followed up initially through the clinic were included in analyses $(\mathrm{n}=1608)$; individuals for which no initial VS identification was attempted through clinic medical records were not included in analyses $(\mathrm{n}=285)$. Individuals considered completely LTFU were excluded from the analysis of LTCassociated risk for premature mortality $(n=100)$. 


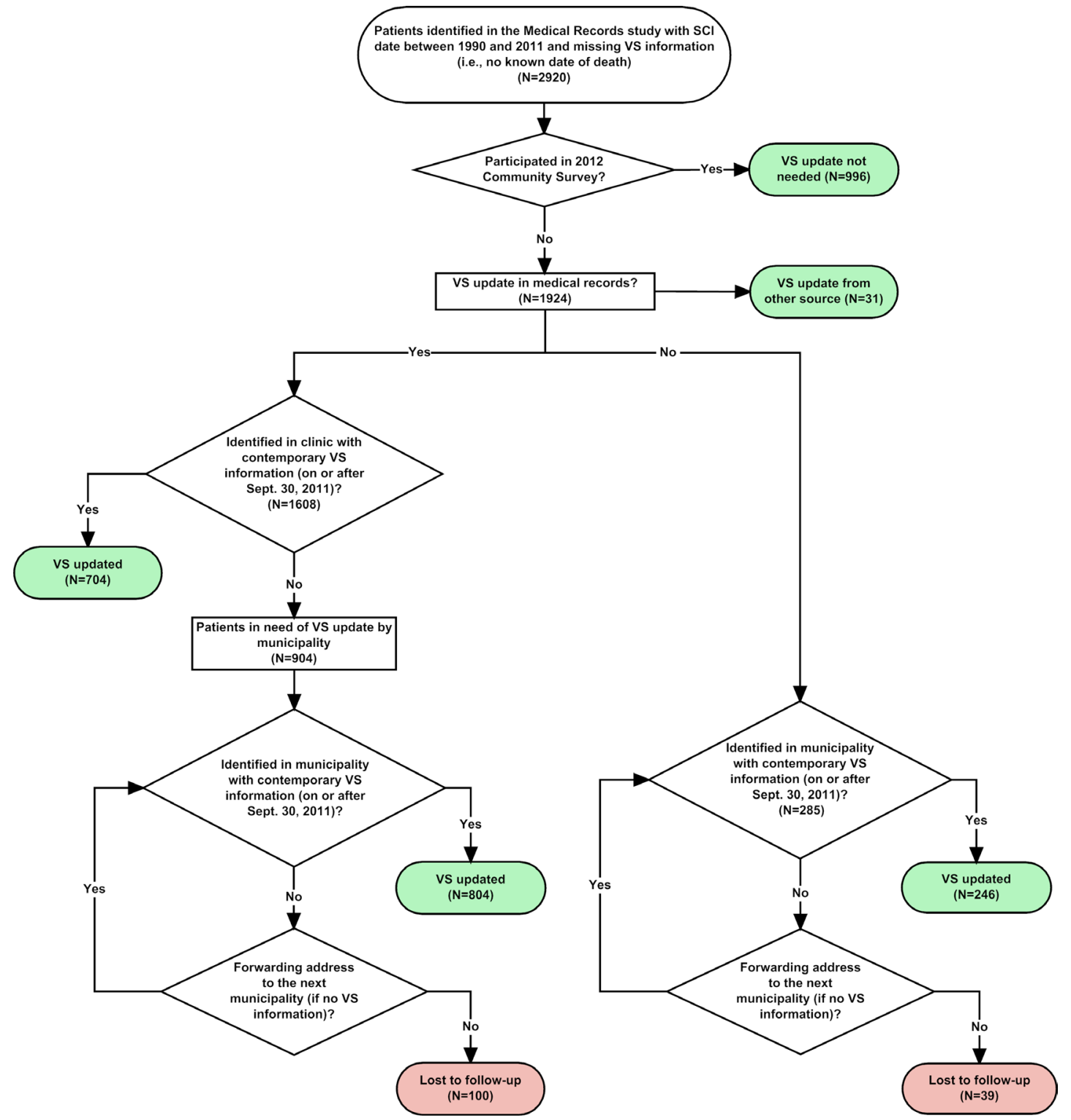

Figure 1 Tracing methodology for vital status ascertainment. 'VS update from other source $(n=31)$ ' includes individuals included within the VS update tracing study but for whom the VS was ascertained from individuals' response to contact by the SwiSCl study centre. SwiSCI, Swiss Spinal Cord Injury cohort study; VS, vital status.

\section{Risk factors for LTC}

Individuals were considered LTC (yes/no) if their last clinic contact was 18 months or more before the population censoring date of the study (30 September 2011). The dichotomous LTC variable was then used as the outcome of interest in a flexible parametric (FP) survival model, for which time-at-risk started with discharge from specialised rehabilitation including an 18-month buffer, and then ended either at the population censoring date (ie, study end - 30 September 2011) or when patients were identified as LTC. ${ }^{16}$ The 18 -month buffer was chosen based on: (A) clinical recommendations for regular, yearly (12-18 months) follow-up outpatient visits and (B) methodological recommendations by Lesko $e t a l^{17}$ to censor at date of study-specific LTFU definition, rather than date of last contact given that the event of interest (mortality) is captured outside of a study encounter. Risk factors for going LTC included in the model were: driving distance to first rehabilitation clinic (driving time of $<30 \mathrm{~min}$ or driving time of $30 \mathrm{~min}$ or more); age at injury; sex; lesion level and completeness of lesion; discharge destination; and specialised rehabilitation clinic attended. Confounders were identified using directed acyclic graphs (DAGs) informed by theory, previous evidence and data availability (online supplementary figure 1). ${ }^{18}$ The calculation of driving distance mimicked calculations previously carried out in the Swiss context, with distance to clinic measured as driving time from last known address to specialised rehabilitation clinic; this was calculated using road network information available from TomTom MultiNet shapefiles (2011) and ArcGIS Network Analyst. ${ }^{19}$ Hospital addresses were identified using data from the Swiss Federal Statistics Office and Federal Office of Topography (swisstopo), 
with coordinates derived using geocoding tools available within the ArcGIS 10.3 suite. Driving duration was estimated in minutes to destination. Driving time to the closest specialised rehabilitation clinic was dichotomised (ie, less than $30 \mathrm{~min}$ driving time vs $30 \mathrm{~min}$ or more driving time). Analyses were stratified according to aetiology of SCI (ie, TSCI or NTSCI). To ensure robustness of risk factor identification, in sensitivity analyses, multiple imputation (MI) using chained equations was used to fill in missing information for completeness of lesion $(n=89)$ - using Rubin's rules for derivation of global point estimates and confidence intervals from 20 imputations. ${ }^{20}$ Bootstrapping (using 1000 replications) was further used to account for potential chance effects due to small sample size.

\section{Influence of LTC on risk of mortality}

An FP survival model was employed to determine the influence of LTC on risk of premature mortality. ${ }^{16}$ For this analysis, time-at-risk started with discharge from specialised rehabilitation (including an 18-month buffer window) and ended with death or administrative censoring due to study end. Given the likely differential force of mortality attributable to ageing with a spinal cord injury, attained age (ie, age at death or study end) was used in place of age at injury. ${ }^{21}$ For those LTC, time-at-risk was partitioned according to pre-LTC and post-LTC based on date of last clinic contact using splitting techniques. This allows for the follow-up time contribution to separate risk sets (ie, time when not LTC and time when LTC) and subsequent calculation of corresponding HRs of risk for mortality (online supplementary figure 2). Similar to the aforementioned analysis, confounders were identified using DAGs (online supplementary figure 3). For multilevel categories, post hoc testing was used to evaluate support for between-level differences. In order to account for potential centre effects, the cluster optioncluster ()—was specified. E-values and associated lower confidence intervals (LCIs) were calculated to assess the robustness of effect estimates in the presence of unmeasured confounding. ${ }^{22}$ Briefly, large E-values would indicate that the unmeasured confounding required would need to be considerable to explain away observed associate. Importantly, small E-values do not demonstrate that no effect exists, only that a weak unmeasured confounder could explain away observed association. ${ }^{23}$

All analyses were conducted using Stata V.14.2 for Windows.

\section{Patient and public involvement}

Any study undertaken using data collected within the context of the SwiSCI cohort study must be first approved by members of the SwiSCI Steering Committee. This committee includes community representatives who are provided the opportunity to give feedback on the study design and outcomes. Therefore, although this research project did not directly involve patients or the public in the analysis and interpretation of results, a committee including patient representatives approved the database and initial study design.

\section{RESULTS}

\section{Descriptive statistics}

Within the SwiSCI Medical Records study, 2920 individuals were injured or suffered a SCI between 1990 and 30 September 2011 and required updated information on their VS. Of these individuals, VS was initially checked in the clinic for $83.4 \%$ ( $n=1608$ ) (figure 1). In $43.8 \%$ of these cases $(n=704)$, contemporary VS information was ascertained through clinic records. Of those individuals for which contemporary VS was not ascertained from clinic records $(\mathrm{n}=904)$, nearly 90\% $(\mathrm{n}=804)$ were identified in municipalities (figure 1). VS was ascertained for $86 \%$ $(n=246)$ of the cases that were checked directly through municipality centres $(\mathrm{n}=285)$ (figure 1$)$. Individuals were considered LTFU if VS could not be ascertained from medical records and/or through municipalities $(n=139)$ (figure 1). Given the observed differences in study participant characteristics according to SCI aetiology-that is, NTSCI or TSCI-all analyses were stratified according to SCI aetiology (table 1).

\section{Risk factors for clinic attrition}

Risk factors associated with going LTC differed across SCI aetiologies (table 2). Persons with an attained TSCI contributed to a total follow-up time of 4700.6 years (average $=5.8$ years), while persons with NTSCI contributed a total follow-up time of 1648.4 person-years (average $=3.3$ years). Among persons with TSCI, younger ages were associated with a lower risk of LTC, with a more than twofold reduction in risk for younger individuals between 16 and 30 years of age (HR=0.44; $95 \%$ CI 0.31 to $0.64)$ and 31 and 45 years of age ( $\mathrm{HR}=0.46 ; 95 \%$ CI 0.32 to 0.67 ) in comparison with 61-75year olds (table 2). Complete lesions in persons with TSCI were associated with a nearly $40 \%$ reduction in risk of going lost to clinic ( $\mathrm{HR}=0.73 ; 95 \%$ CI 0.55 to 0.98$)$. No difference in risk was observed according to lesion level (table 2). In the group of persons with TSCI, a tendency for a reduction in risk of nearly $30 \%$ was observed for more recent injuries versus injuries incurred between 1990 and 1999 (HR=0.79; 95\% CI 0.60 to 1.03 ); this remained stable in sensitivity analyses of MI and bootstrapped results. Extended driving time to the clinic of first rehabilitation was associated with an increase in risk of LTC for both persons with TSCI and NTSCI (HR $=1.39 ; 95 \%$ CI 1.06 to 1.81 and $\mathrm{HR}=1.59 ; 95 \%$ CI 1.12 to 2.26 , respectively) (table 2 ).

\section{LTC as a risk factor for premature mortality}

A total of 1507 cases contributed to 10827.7 personyears at risk (average $=7.2$ years), with 7727.2 personyears attributed to persons with TSCI (average $=8.6$ years; deaths $=103$ ), and 3.100 .5 person-years to persons with NTSCI (average $=5.1$ years; deaths $=160$ ). After controlling for current age, lesion level, completeness 


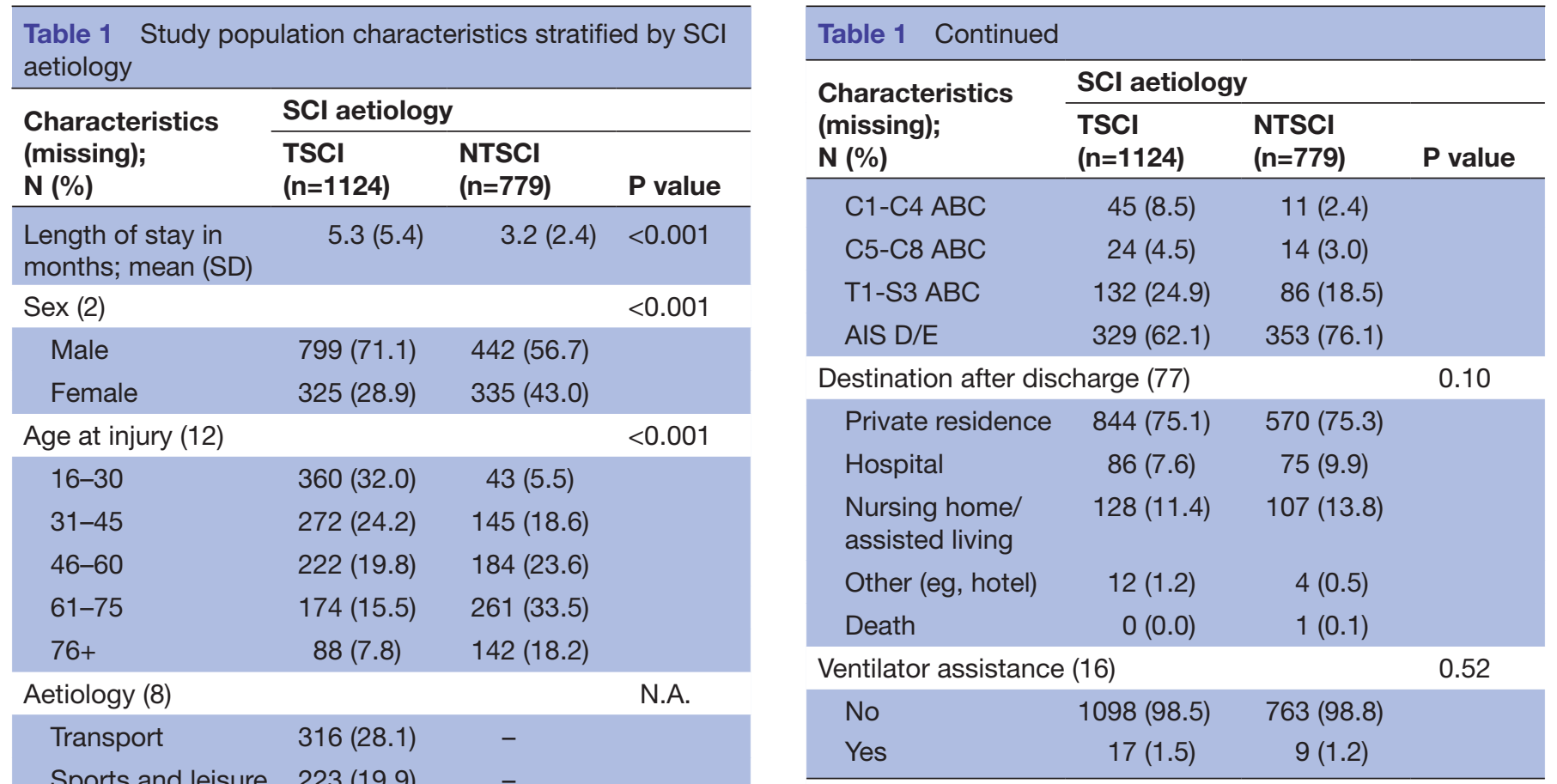

$\begin{array}{lcc}\text { Sports and leisure } & 223(19.9) & - \\ \text { Falls } & 444(39.5) & - \\ \text { Other - TSCI } & 140(12.5) & - \\ \text { Tumour } & - & 202(26.2) \\ \text { Inflammation } & - & 26(3.4) \\ \text { Bleeding } & - & 45(5.8) \\ \text { Infection } & - & 37(4.8) \\ \text { Other }-\mathrm{NTSCl} & - & 462(59.8)\end{array}$

$\begin{array}{lccc}\text { Level of SCI (13) } & & & <0.001 \\ \text { Tetra } & 614(55.0) & 456(59.0) & \\ \text { Para } & 415(37.2) & 217(28.1) & \\ \text { Cauda equina } & 88(7.9) & 100(12.9) & \\ \text { Completeness (110) } & & & <0.001 \\ \text { Incomplete } & 795(74.6) & 670(92.2) & \\ \text { Complete } & 271(25.4) & 57(7.8) & \end{array}$

Lesion level and completeness

(267)

\begin{tabular}{lccc}
$\begin{array}{l}\text { Paraplegia, } \\
\text { incomplete }\end{array}$ & $382(38.4)$ & $388(60.5)$ & \\
$\begin{array}{l}\text { Paraplegia, } \\
\text { complete }\end{array}$ & $215(21.6)$ & $44(6.7)$ & \\
$\begin{array}{l}\text { Tetraplegia, } \\
\text { incomplete }\end{array}$ & $343(34.5)$ & $202(31.5)$ & \\
$\begin{array}{l}\text { Tetraplegia, } \\
\text { complete }\end{array}$ & $55(5.5)$ & $7(1.1)$ & \\
$\begin{array}{l}\text { ASIA score (677) } \\
\text { AIS A }\end{array}$ & $168(24.6)$ & $36(6.6)$ & \\
AIS B & $66(9.6)$ & $49(9.0)$ & \\
AIS C & $120(17.7)$ & $105(19.3)$ & \\
AIS D/E & $329(48.1)$ & $353(65.0)$ & \\
Injury severity (909) & & & $<0.001$ \\
\hline
\end{tabular}

Twenty-one individuals missing information on $\mathrm{SCl}$ aetiology. AIS, ASIA Impairement Scale; ASIA, American Spinal Injury Association; NTSCI, non-traumatic spinal cord injury; SCI, spinal cord injury; TSCI, traumatic spinal cord injury.

and sex, LTC was associated with an increased risk of mortality ( $\mathrm{HR}=3.62 ; 95 \%$ CI 2.18 to 6.02 ) for persons with TSCI (figure 2), corresponding to an E-value of 6.70 (LCI=3.78). This association remained when including MI data (HR=3.07; 95\% CI 2.29 to 4.13), as well as when using bootstrapping ( $\mathrm{HR}=3.59$; 95\% CI 2.16 to 6.00). In comparison with TSCI, no association between LTC and risk of mortality was evident for persons with NTSCI (E-value=1.88; LCI=1.00). Sensitivity analyses using MI or bootstrapping gave similar results. In a secondary analysis investigating distance to rehabilitation as a risk factor for mortality, there was no evidence to suggest any association for persons with TSCI ( $\mathrm{HR}=1.34,95 \%$ CI 0.76 to 2.37). However, for persons with NTSCI, a heightened risk of mortality was observed for those living $30 \mathrm{~min}$ or further from specialised rehabilitation ( $\mathrm{HR}=1.51,95 \%$ CI 1.02 to 2.22), corresponding to an E-value of 2.39 (LCI=1.16).

\section{DISCUSSION}

\section{Summary}

This is the first study to our knowledge that has investigated LTC in the context of spinal cord injury, and the first to investigate the consequences of LTC in the broader Swiss setting. The key insight emerging from this study is that LTC in persons living with TSCI may be indicative of an elevated risk of death. Characteristics of persons with TSCI who are at higher risk to go LTC include: older age at injury, incomplete lesion, discharge destination to hospital and a driving time of more than 30 minutes to specialised rehabilitation 


\begin{tabular}{|c|c|c|}
\hline & TSCI & NTSCI \\
\hline \multicolumn{3}{|l|}{ Sex } \\
\hline Male & Reference & Reference \\
\hline Female & $1.00(0.77$ to 1.30$)$ & 1.45 (1.08 to 1.94$)$ \\
\hline \multicolumn{3}{|c|}{ Age at injury (years) } \\
\hline $16-30$ & $0.44(0.31$ to 0.64$)$ & 0.95 (0.50 to 1.82$)$ \\
\hline $31-45$ & $0.46(0.32$ to 0.67$)$ & 0.43 (0.26 to 0.70$)$ \\
\hline $46-60$ & 0.51 (0.35 to 0.76$)$ & 0.71 (0.48 to 1.03$)$ \\
\hline $61-75$ & Reference & Reference \\
\hline$\geq 76$ & $1.62(1.02$ to 2.58$)$ & 1.34 (0.90 to 2.01$)$ \\
\hline \multicolumn{3}{|l|}{ Decade } \\
\hline 1990-1999 & Reference & Reference \\
\hline 2000-2011 & 0.79 (0.60 to 1.03$)$ & 1.26 (0.88 to 1.81$)$ \\
\hline \multicolumn{3}{|l|}{ Lesion level } \\
\hline Paraplegia & Reference & Reference \\
\hline Tetraplegia & $0.98(0.76$ to 1.27$)$ & 0.96 (0.71 to 1.31$)$ \\
\hline \multicolumn{3}{|c|}{ Lesion completeness } \\
\hline Incomplete & Reference & Reference \\
\hline Complete & 0.73 (0.55 to 0.98$)$ & 0.98 (0.49 to 1.97$)$ \\
\hline \multicolumn{3}{|c|}{ Distance to first rehabilitation clinic } \\
\hline$<30 \min$ & Reference & Reference \\
\hline$>30 \mathrm{~min}$ & 1.39 (1.06 to 1.81$)$ & 1.59 (1.12 to 2.26$)$ \\
\hline
\end{tabular}

Rehabilitation centre

\begin{tabular}{|c|c|c|}
\hline A & Reference & Reference \\
\hline B & 1.44 (0.98 to 2.10$)$ & 1.38 (0.86 to 2.20$)$ \\
\hline C & 1.36 (0.98 to 1.90$)$ & 1.14 (0.79 to 1.64$)$ \\
\hline $\mathrm{D}$ & 3.89 (2.58 to 5.85$)$ & 0.96 (0.28 to 3.21$)$ \\
\hline \multicolumn{3}{|c|}{ Discharge destination } \\
\hline Home & Reference & Reference \\
\hline Hospital & 1.87 (1.17 to 2.98$)$ & 1.38 (0.70 to 2.74$)$ \\
\hline $\begin{array}{l}\text { Nursing/ } \\
\text { assisted living/ } \\
\text { group home }\end{array}$ & $1.12(0.78$ to 1.61$)$ & 1.43 (0.94 to 2.19$)$ \\
\hline Other & 1.50 (0.55 to 4.08$)$ & 2.51 ( 0.34 to 18.40 \\
\hline
\end{tabular}

Names of rehabilitation facilities (A, B, C and D) refer to the four specialised rehabilitation centres within Switzerland with eligible data for the present study. Twenty-one cases excluded due to missing information on $\mathrm{SCl}$ aetiology.

$\mathrm{NTSCl}$, non-traumatic spinal cord injury; SCI, spinal cord injury; $\mathrm{TSCl}$, traumatic spinal cord injury.

clinic. In addition, for individuals with TSCI, going lost to clinic was associated with a nearly fourfold higher risk of mortality. For individuals with an attained NTSCI, only gender and driving time to specialised rehabilitation influenced risk of LTC. However, in contrast to TSCI, driving time was associated with a higher risk of mortality for persons with NTSCI.

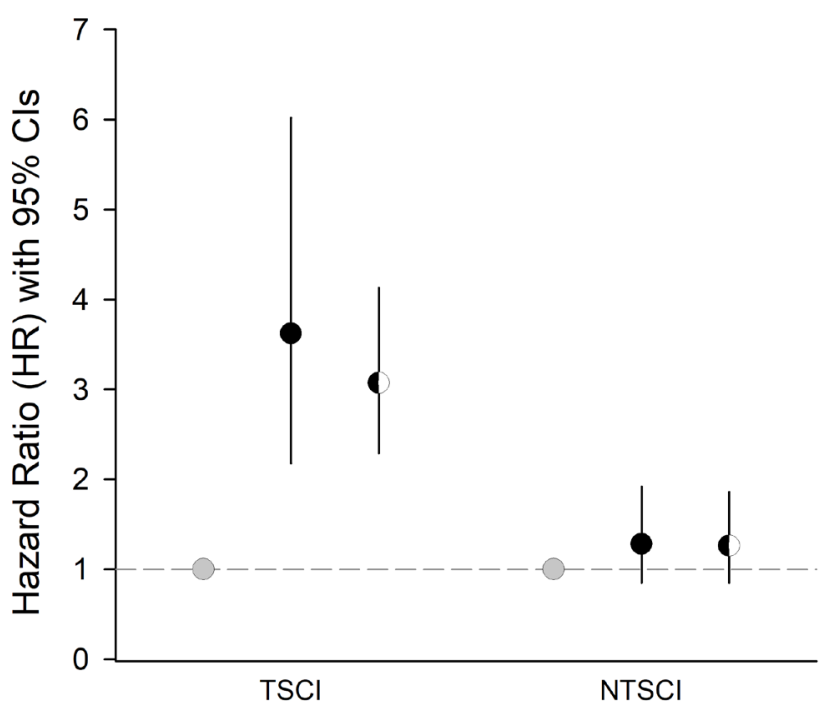

\section{Lost to clinic (LTC)}

Figure 2 Risk of mortality based on lost to clinic (LTC) status. Grey-filled circles represent the reference group, 'not LTC', while black-filled circles indicate the HR for those 'LTC'. Semifilled circles represent HRs based on multiple imputation. Vertical black lines correspond to estimated 95\% Cls. NTSCI, traumatic spinal cord injury; TSCl, traumatic spinal cord injury.

\section{Importance of continuity of care}

Spinal cord injury is a chronic and serious medical condition that can result in severe physical and mental disorders often requiring specialised acute and follow-up care. A recent survey on morbidity in the Swiss SCI community showed that individuals had on average seven concomitant health conditions or symptoms. ${ }^{24}$ The most commonly reported physical problems included chronic pain, spasticity, sexual dysfunction, bowel dysfunction, bladder dysfunction, urinary tract infection and sleep problems, with 3-month prevalence ranging between $74 \%$ and $59 \% .{ }^{24}$ In addition, prevalence of depression was estimated at $15 \% .{ }^{24}$ While most of these health issues emerge on or shortly after injury, they can also develop in the months following or up to 1 year post-injury. Accordingly, SCI has been associated with substantially elevated all-cause and cause-specific mortality, including mortality due to cardiac or ischaemic heart disease, septicaemia, specific infections (eg, urinary tract or respiratory infection) and suicide. ${ }^{13}{ }^{25}$ These findings underscore the importance of regular, life-long follow-up as part of specialised medical care to facilitate early diagnosis and effective prevention and treatment of health issues.

Given the complex and chronic nature of spinal cord injuries, a plausible mechanism driving the elevated mortality in persons LTC could be discontinuity of care, with LTC being indicative of discontinued care. Supporting this, previous research has found that improved continuity of care is associated with lower mortality rates and improved health outcomes. ${ }^{26-28}$ Although few SCI-specific studies 
have investigated continuity of care on health outcomes, one contemporary study found that persons with continuity of care were less likely to partake in polypharmacy. ${ }^{29}{ }^{30}$ To this effect, to improve survival outcomes, regular, life-long specialised care is critical for the longterm management of secondary health conditions (eg, cardiovascular disease, urinary tract infections, respiratory infections and pressure ulcers) associated with premature mortality. ${ }^{13}$ However, current evidence suggests inadequate management and support of secondary health conditions. For example, a 2012 study by Guilcher et al ${ }^{31}$ found that of the emergency department (ED) visits by persons with SCI, nearly $20 \%$ were potentially preventable and roughly three quarters of those visits were due to SCI-specific conditions such as urinary tract infections or pneumonia. Although the reason behind ED usage as a primary care facility is unknown, it may be due to limited access to specialised services given that rurality has been associated with higher ED usage. ${ }^{31}{ }^{32}$

In a recent study that investigated satisfaction in care for persons living with SCI in the Swiss community, individuals were found to be less satisfied with availability, quality and fulfilment of care and care needs with increased distance from specialised rehabilitation centres. ${ }^{33}$ In this study, among individuals with TSCI, driving time to specialised rehabilitation was associated with LTC, although not the subsequent risk of premature mortality. A potential explanation for this could be that LTC mediates the association between driving time and premature mortality, which would suggest that it is not access in general that influences mortality outcomes and other adverse health outcomes, but rather access and continued care in specialised rehabilitation facilities. In support of this, previous chronic disease and other SCI-specific literature similarly points towards the positive influence of specialised rehabilitation on health outcomes. ${ }^{34-36}$ However, in contrast to TSCI, no association was found for individuals with an attained NTSCI between LTC and mortality, although there was an apparent association between driving time and subsequent risk of mortality. This could be indicative of potentially differing patterns of healthcare utilisation due to the impact of ageing with a SCI and subsequent requirements for long-term continuation of care. It is also important to note that no evidence exists that provides a concrete comparison as to whether LTC from a specialised rehabilitation centre is more important than LTC from generalised care, such as a family physician. Research addressing the added value of specialised rehabilitation in improving consequences of LTC is thus needed.

Unfortunately, causal inference on the relevancy of discontinuity of care with respect to mortality is limited due to lack of direct, critical evidence regarding the extent to which improved continuity of care may improve health outcomes and mortality. Thus, while observational studies are important, and often the only ethical solution, for contributing to evidence-based decisions and causal inference, given the secondary, post-study design nature of the present study, results from this study may be subject to unmeasured confounding factors that affect both clinic attendance and secondary health conditions/morbidity. ${ }^{37}$ For example, secondary health conditions may influence an individual's propensity towards continued care-including the type of clinic visited (ie, specialised vs not specialised) - as well as an individual's risk of mortality. ${ }^{38}$ However, the E-value estimated in this study for persons with TSCI demonstrates that to explain away the observed association between LTC and risk of mortality, the unmeasured confounder would need to be associated with both LTC and risk of mortality by a nearly sevenfold risk ratio. Nevertheless, for causal inference pertaining to the role of LTC on subsequent risk of mortality, intervention studies designed to specifically target this research question and sufficiently account for potential confounders are needed, particularly given the weak evidence for persons with NTSCI.

\section{Methodological aspect}

This study draws attention to the use of routine follow-up data in evaluating survival and life expectancy in people living with a chronic, physical impairment such as spinal cord injury. Similar to findings in other contexts, such as HIV or cancer, incomplete ascertainment of VS commonly induces underestimation of mortality given that individuals with a higher propensity for premature mortality are more likely to go LTC. ${ }^{239}$ Furthermore, it is very likely that those completely LTFU (ie, no vital status information could be ascertained) have an elevated risk of mortality. The tracing methodology presented in this study provides a feasible approach for garnering additional information on LTC patterns and the associated risk of mortality; VS could also be obtained through linkage with national death registries, if available. ${ }^{40}$ Such information can be used to inform future analyses aimed at attenuating the potential bias on estimates of mortality or other clinical outcomes, for example, through sensitivity analysis, inverse probability weighting, MI or pattern mixture modelling. ${ }^{240}$

\section{Study limitations}

The data used for this study include augmented information from a follow-up study meant to ascertain detailed vital status information, including date of death, and for some cases date of last clinic contact. However, when identifying date of last clinic contact, only the original specialised rehabilitation clinic was checked; that is, we did not cross-reference cases between clinics. Nonetheless, switching between specialised rehabilitation institutions for follow-up care is relatively rare and unlikely to account for differential mortality related to LTC. Additionally, during the tracing effort, in the situation that a new address was identified, databases were updated; thus, the contemporary address was used for all distance calculations. Another potential limitation is that to be eligible for analyses, it was required that VS first be checked in medical records. Given that clinic records were checked systematically in only three of the specialised rehab 
clinics, there is a potential for unmeasured confounding between patient variables and risk of LTC, which could subsequently affect the association between LTC and risk of mortality. However, exclusion of specialised rehabilitation clinics that did not undergo a systematic check of medical records-apart from decade, which was no longer associated with risk of LTC-did not modify overall conclusions. In addition to this, as mentioned above, another potential, unmeasured confounder are secondary health conditions. Unfortunately, information on secondary health conditions was not collected in the SwiSCI Medical Records. However, E-value estimates demonstrate that such an unmeasured confounder would need to be very strongly associated with both the exposure and outcome in order to completely obviate the observed association. Finally, the SwiSCI study only includes individuals admitted to one of the SCI-specialised rehabilitation centres in Switzerland; differences between patient populations have been evidenced; therefore, these results may not be generalisable to the entire Swiss SCI population. ${ }^{10}$

Due to data limitations, it was not possible to provide an estimate of the underestimation of, for example, 1-year mortality; such an estimate would require mortality information on individuals LTFU. In the study by Brinkhof $e t$ $a l,{ }^{2}$ authors used a pattern-mixture model to impute the missing survival times for individuals LTFU. Future analyses using the SwiSCI data could use a similar approach to account for such survival bias. However, given that unmanaged or ineffectively treated HIV infection nearly always leads to certain death, the natural force of mortality in SCI is considerably less. We would therefore expect a much lower relative increase in mortality if including SCI patients LTFU.

\section{Conclusion}

The present study provides evidence that going LTC is associated with poorer survival prospects among individuals with TSCI. Patterns of LTC may help illuminate risk factors for attrition from specialised care and consequently aid in identifying higher risk groups requiring targeted care to improve health outcomes and reduce premature mortality. ${ }^{5}$ However, further research is needed to determine whether improving continuation of care in specialised rehabilitation centres will lead to better health outcomes.

\author{
Author affiliations \\ ${ }^{1}$ Swiss Paraplegic Research, Nottwil, Switzerland \\ ${ }^{2}$ Centre INSERM U1219, CIC 1401-EC, Institut de Santé Publique, d'Epidémiologie \\ et de Développement (ISPED), Bordeaux School of Public Health, University of \\ Bordeaux, Bordeaux, France \\ ${ }^{3}$ Swiss Paraplegic Centre, Nottwil, Switzerland \\ ${ }^{4}$ REHAB Basel, Basel, Switzerland \\ ${ }^{5}$ Clinique Romande de Réadaptation, Sion, Switzerland \\ ${ }^{6}$ Balgrist University Hospital, Zürich, Switzerland \\ ${ }^{7}$ Department of Health Sciences and Medicine, University of Lucerne, Luzern, \\ Switzerland
}

Acknowledgements This study has been financed in the framework of the Swiss Spinal Cord Injury Cohort Study (SwiSCl; www.swisci.ch), supported by the Swiss
Paraplegic Foundation. The members of the SwiSCI Steering Committee are: Xavier Jordan, Fabienne Reynard (Clinique Romande de Réadaptation, Sion); Michael Baumberger, Hans Peter Gmünder (Swiss Paraplegic Center, Nottwil); Armin Curt and Martin Schubert (University Clinic Balgrist, Zürich); Margret Hund-Georgiadis and Kerstin Hug (REHAB Basel, Basel); Urs Styger (Swiss Paraplegic Association, Nottwil); Daniel Joggi (Swiss Paraplegic Foundation, Nottwil); NN (representative of persons with SCI); Nadja Münzel (Parahelp, Nottwil); Mirjam Brach and Gerold Stucki (Swiss Paraplegic Research, Nottwil); and Armin Gemperli (SwiSCl Coordination Group at Swiss Paraplegic Research, Nottwil).

Contributors JDC and MWGB were responsible for conceptual framing of the present study. MWGB further provided statistical support as well as critical feedback on manuscript content. IEE-H, KH, XJ and MS provided clinical support and feedback of the present manuscript, as well as support in data collection at their respective clinics. JDC was responsible for all analyses, drafting and finalisation of the present manuscript.

Funding This study has been financed in the framework of the SwiSCl (www. swisci.ch).

Competing interests None declared.

Patient consent for publication Not required.

Ethics approval The SwiSCI cohort study has been approved by local ethics committees (reference numbers: 1008 (Luzern); 37/11 (Basel); CCVEM 015/11 (Valais); 2012-0049 (Zürich)).

Provenance and peer review Not commissioned; externally peer reviewed.

Data availability statement Data are available on reasonable request. The data used in this study are available from the SwiSCl Study Center at Swiss Paraplegic Research, Nottwil. These data are not publicly available; therefore, restrictions apply as to its availability. However, data are available from the authors on reasonable request and with permission of the SwiSCI Study Center at Swiss Paraplegic Research.

Open access This is an open access article distributed in accordance with the Creative Commons Attribution Non Commercial (CC BY-NC 4.0) license, which permits others to distribute, remix, adapt, build upon this work non-commercially, and license their derivative works on different terms, provided the original work is properly cited, appropriate credit is given, any changes made indicated, and the use is non-commercial. See: http://creativecommons.org/licenses/by-nc/4.0/.

ORCID iD

Jonviea D Chamberlain http://orcid.org/0000-0002-9515-1076

\section{REFERENCES}

1 Chatfield MD, Brayne CE, Matthews FE. A systematic literature review of attrition between waves in longitudinal studies in the elderly shows a consistent pattern of dropout between differing studies. $J$ Clin Epidemiol 2005;58:13-19.

2 Brinkhof MWG, Spycher BD, Yiannoutsos C, et al. Adjusting mortality for loss to follow-up: analysis of five art programmes in sub-Saharan Africa. PLoS One 2010;5:e14149.

3 Kristman V, Manno M, Côté P. Loss to follow-up in cohort studies: how much is too much? Eur J Epidemiol 2004;19:751-60.

4 WHO. International perspectives on spinal cord injury. Malta: World Health Organization, 2013.

5 Chamberlain JD, Meier S, Mader L, et al. Mortality and longevity after a spinal cord injury: systematic review and meta-analysis. Neuroepidemiology 2015;44:182-98.

6 Hagen EM, Lie SA, Rekand T, et al. Mortality after traumatic spinal cord injury: 50 years of follow-up. J Neurol Neurosurg Psychiatry 2010;81:368-73.

7 Middleton JW, Dayton A, Walsh J, et al. Life expectancy after spinal cord injury: a 50-year study. Spinal Cord 2012;50:803-11.

8 Kuehni CE, Rueegg CS, Michel G, et al. Cohort profile: the Swiss childhood cancer Survivor study. Int J Epidemiol 2012;41:1553-64.

9 Chamberlain JD, Deriaz O, Hund-Georgiadis M, et al. Epidemiology and contemporary risk profile of traumatic spinal cord injury in Switzerland. Inj Epidemiol 2015;2:28.

10 Chamberlain JD, Ronca E, Brinkhof MW. Estimating the incidence of traumatic spinal cord injuries in Switzerland: using administrative data to identify potential coverage error in a cohort study. Swiss Med Wkly 2017;147:w14430.

11 Post MWM, Brinkhof MWG, von Elm E, et al. Design of the Swiss spinal cord injury cohort study. Am J Phys Med Rehabil 2011;90:S5-16. 
12 Brinkhof MWG, Fekete C, Chamberlain JD, et al. Swiss national community survey on functioning after spinal cord injury: protocol, characteristics of participants and determinants of non-response. $J$ Rehabil Med 2016;48:120-30.

13 Chamberlain JD, Buzzell A, Gmünder HP, et al. Comparison of allcause and cause-specific mortality of persons with traumatic spinal cord injuries to the general Swiss population: results from a national cohort study. Neuroepidemiology 2019;52:205-13.

14 Spreyermann R, Lüthi H, Michel F, et al. Long-Term follow-up of patients with spinal cord injury with a new ICF-based tool. Spinal Cord 2011;49:230-5.

15 DeVivo M, Chen Y, Krause J, et al. Trends in age-adjusted causespecific mortality rates after spinal cord injury. Top Spinal Cord Inj Rehabil 2012;214.

16 Royston P, Lambert PC. Flexible Parametric Survival Analysis Using Stata: Beyond the Cox Model. Stata Press, 2011.

17 Lesko CR, Edwards JK, Cole SR, et al. When to Censor? Am J Epidemiol 2018;187:623-32.

18 Greenland S, Pearl J, Robins JM. Causal diagrams for epidemiologic research. Epidemiology 1999;10:37-48

19 Berlin C, Panczak R, Hasler R, et al. Do acute myocardial infarction and stroke mortality vary by distance to hospitals in Switzerland? results from the Swiss national cohort study. BMJ Open 2016;6:e013090.

20 Rubin DB. Multiple imputation for survey nonresponse. New York: Wiley, 1987.

21 Chamberlain JD, Gmünder HP, Hug K, et al. Differential survival after traumatic spinal cord injury: evidence from a multi-center longitudina cohort study in Switzerland. Spinal Cord 2018:56:920-30.

22 VanderWeele TJ, Ding P. Sensitivity analysis in observational research: introducing the E-Value. Ann Intern Med 2017;167:268-74.

23 VanderWeele TJ, Mathur MB, Ding P. Correcting misinterpretations of the E-ValueCorrecting misinterpretations of the E-Value. Annals of internal medicine 2019;170:131-2.

24 Brinkhof MWG, Al-Khodairy A, Eriks-Hoogland I, et al. Health conditions in people with spinal cord injury: contemporary evidence from a population-based community survey in Switzerland. J Rehabil Med 2016;48:197-209.

25 Buzzell A, Chamberlain JD, Eriks-Hoogland I, et al. All-Cause and cause-specific mortality following non-traumatic spinal cord injury: evidence from a population-based cohort study in Switzerland. Spinal Cord 2020;58:157-64.

26 Pereira Gray DJ, Sidaway-Lee K, White E, et al. Continuity of care with Doctors-a matter of life and death? A systematic review of continuity of care and mortality. BMJ Open 2018;8:e021161.
27 Weir DL, McAlister FA, Majumdar SR, et al. The interplay between continuity of care, multimorbidity, and adverse events in patients with diabetes. Med Care 2016;54:386-93.

28 Hoertel N, Limosin F, Leleu H. Poor longitudinal continuity of care is associated with an increased mortality rate among patients with mental disorders: results from the French National health insurance reimbursement database. Eur Psychiatry 2014;29:358-64.

29 Guilcher SJT, Hogan M-E, Calzavara A, et al. Prescription drug claims following a traumatic spinal cord injury for older adults: a retrospective population-based study in Ontario, Canada. Spinal Cord 2018;56:1059-68.

30 Bloemen-Vrencken JHA, de Witte LP, Post MWM. Follow-Up care for persons with spinal cord injury living in the community: a systematic review of interventions and their evaluation. Spinal Cord 2005:43:462-75.

31 Guilcher SJT, Craven BC, Calzavara A, et al. Is the emergency department an appropriate substitute for primary care for persons with traumatic spinal cord injury? Spinal Cord 2013;51:202-8.

32 Munce SEP, Guilcher SJT, Couris CM, et al. Physician utilization among adults with traumatic spinal cord injury in Ontario: a population-based study. Spinal Cord 2009:47:470-6.

33 Ronca E, Scheel-Sailer A, Koch HG, et al. Satisfaction with access and quality of healthcare services for people with spinal cord injury living in the community. J Spinal Cord Med 2020;43:111-21.

34 Parent S, Barchi S, LeBreton M, et al. The impact of specialized centers of care for spinal cord injury on length of stay, complications, and mortality: a systematic review of the literature. J Neurotrauma 2011;28:1363-70.

35 Cheng CL, Plashkes T, Shen T, et al. Does specialized inpatient rehabilitation affect whether or not people with traumatic spinal cord injury return home? J Neurotrauma 2017;34:2867-76.

36 Kinchen KS, Sadler J, Fink N, et al. The timing of specialist evaluation in chronic kidney disease and mortality. Ann Intern Med 2002:137:479-86.

37 Hernán M. The C-Word: the more we discuss it, the less dirty it sounds. Am J Public Health 2018;108:625-6.

38 Krause JS, Saunders LL, Health SLL. Health, secondary conditions, and life expectancy after spinal cord injury. Arch Phys Med Rehabil 2011;92:1770-5.

39 Spiers S, Oral E, Fontham ETH, et al. Modelling attrition and Nonparticipation in a longitudinal study of prostate cancer. BMC Med Res Methodol 2018;18:60.

40 Schomaker M, Gsponer T, Estill J, et al. Non-ignorable loss to followup: correcting mortality estimates based on additional outcome ascertainment. Stat Med 2014;33:129-42. 